\title{
Sum Rate Optimization by Spatial Precoding for a Multiuser MIMO DFT-Precoded OFDM Uplink
}

\section{Hanguang Wu, ${ }^{1}$ Thomas Haustein (EURASIP Member), ${ }^{2}$ Eduard Axel Jorswieck (EURASIP Member), ${ }^{3}$ and Peter Adam Hoeher ${ }^{4}$}

\author{
${ }^{1}$ mimoOn GmbH, Bismarckstraße 120, 47057 Duisburg, Germany \\ ${ }^{2}$ Fraunhofer-Institute for Telecommunications, Heinrich-Hertz-Institute, Einsteinufer 37, 10587 Berlin, Germany \\ ${ }^{3}$ Communications Laboratory, Dresden University of Technology, 01062 Dresden, Germany \\ ${ }^{4}$ Faculty of Engineering, University of Kiel, Kaiserstraße 2, 24143 Kiel, Germany
}

Correspondence should be addressed to Hanguang Wu, wuhanguang@gmail.com

Received 15 October 2010; Revised 31 January 2011; Accepted 10 February 2011

Academic Editor: Robert Fischer

Copyright () 2011 Hanguang Wu et al. This is an open access article distributed under the Creative Commons Attribution License, which permits unrestricted use, distribution, and reproduction in any medium, provided the original work is properly cited.

\begin{abstract}
By means of DFT-precoding, the PAPR of OFDM waveforms can be reduced. DFT-precoding has been proposed for uplink transmission in various future wireless communication systems. In this work, we consider DFT-precoding combined with spatial precoding for the uplink of multiuser MIMO OFDM systems. An efficient algorithm is developed to optimize the spatial precoder aiming at maximization of the system sum rate subject to individual power constraints of the users and maintenance of the low PAPR property for one user. Potential gains are shown compared to other popular precoding methods in 3GPP-LTE uplink scenarios.
\end{abstract}

\section{Introduction}

Over the last years, the demand for high data rates has increased significantly. This has led to the use of wider transmission bandwidth and MIMO (Multiple-Input MultipleOutput) techniques. In a cellular environment and especially at large transmission bandwidth, the channel between the antennas at the transmitter and receiver becomes increasingly frequency selective. OFDM (Orthogonal FrequencyDivision Multiplex) with its multiple access scheme OFDMA (Orthogonal Frequency-Division Multiple Access) is considered as a strong candidate for wideband transmission due to its robustness against frequency selective fading and lowcomputational complexity for channel equalization using frequency domain equalization (FDE) [1]. However, a major drawback of $\operatorname{OFDM}(\mathrm{A})$ is that its transmit waveform has a high peak-to-average power ratio (PAPR). In order to avoid the transmitted signal going into the nonlinear region of the power amplifier, the mean input power has to be limited or backed off to a certain value roughly corresponding to the PAPR (in decibel) of the transmitted waveform. Therefore, high PAPR is power inefficient, especially problematic for cell edge users to be able to overcome the large path loss to reach their serving base station (BS) in uplink transmission.

Various PAPR reduction techniques have been proposed for OFDM systems. A good overview of those techniques is addressed in [2] and the references therein. Among others, DFT-precoding is an attractive solution without requiring any additional signalling overhead. Especially, with loworder modulation schemes like BPSK and QPSK, significantly lower PAPR compared to that of OFDM without precoding is possible [3]. Recently, multiple access schemes based on DFT-precoded OFDM(A) are adopted for uplink transmission in various future mobile communication systems. For example, the 3rd Generation Partnership Project (3GPP) employs DFT-precoded OFDMA with localized subcarrier allocation (LFDMA) [4] for the Long-Term Evolution (LTE) uplink. The localized subcarrier mapping constraint imposed on DFT-precoded OFDMA systems essentially produces a single-carrier waveform which has inherently lower PAPR than that of $\operatorname{OFDM}(\mathrm{A})$ [5]. This structure is also referred to as single-carrier FDMA (SC-FDMA) [6]. 
An alternative possibility to produce a single-carrier waveform is to equidistantly allocate the subcarriers over the entire bandwidth in DFT-precoded OFDMA systems [7]. This subcarrier mapping is also known as interleaved FDMA (IFDMA) [8]. Another variant of DFT-precoded OFDMA using regularly interleaved blocks of subcarriers is denoted as block-IFDMA (B-IFDMA), which provides robustness to frequency offsets at the expense of increased PAPR compared to IFDMA [9] while still having lower PAPR than $\operatorname{OFDM}(\mathrm{A})$ waveforms [10]. This structure has been proposed for nonadaptive uplink transmission in the European Union (EU) 4G research project WINNER [11].

Let us consider the uplink of a multiuser MIMO-OFDM system. If channel state information is available at both the transmitter and receiver, it is known that the optimal precoding matrix applied at the transmitter in terms of asymptotically (The only loss is due to the use of cyclic prefix in OFDM systems, however, the effect becomes negligible when the transmit block size is large. In our discussion, this loss is not considered for simplicity.) achieving the multiple access channel (MAC) capacity can be found efficiently by convex optimization [12]. Generally, the resulting optimal precoding matrices applied to different subcarriers are not the same, as different subcarriers do not experience the same MIMO MAC channel. In order to reduce the signalling overhead to inform the user equipments (UEs) about the subcarrier specific precoding matrix, it is beneficial to apply only one linear precoding matrix for a number of adjacent subcarriers and a number of consecutive OFDM symbols, so-called a chunk [13] or resource block. By applying MMSE successive interference cancellation (SIC) for each subcarrier at the receiver, maximization of the weighted sum rate of MIMO-OFDM MAC is studied in [14], where the problem is formulated under the assumption of individual user power constraints and only one linear precoding matrix applied for a chunk.

In this work, we consider DFT-precoding to be applied to uplink multiuser MIMO OFDM systems as a means to reduce the PAPR of the transmit waveform. For practical interest, we assume that a simple linear zero forcing (ZF) MIMO equalizer is performed on each subcarrier at the receiver to separate the data streams from different users. We propose an algorithm to optimize the spatial precoder aiming at maximization of the system sum rate subject to individual power constraints of the users and maintenance of the low PAPR property of the single-carrier transmit waveform for at least one user. The rest of the paper is organized as follows. Section 2 describes the system model and problem formulation. Section 3 discusses the proposed spatial precoder optimization algorithm and the associated implementation issues. Simulation results are presented in Section 4. Conclusions are drawn in Section 5. Finally Section 6 discusses the open problems and future work.

\section{System Model}

We consider an SC-FDMA uplink with two UEs, each having two antennas and the BS also equipped with two antennas. The generalization to the case with multiple antennas and more than two UEs is possible, which will be discussed later. The block diagram of the system setup is shown in Figure 1. The transmitted data streams $d_{u, 1}, \ldots, d_{u, N}$ of UE $u$ are transformed to the frequency domain via an $N$ point DFT and the DFT output $x_{u, 1}, \ldots, x_{u, N}$ is linear precoded by $\mathbf{v}_{u, 1}, \ldots, \mathbf{v}_{u, N}$, respectively. We assume equal power of the transmitted signal, that is, $E\left\{d_{u, n} d_{u, n}^{*}\right\}=P_{\text {total }, u} / N$, where $P_{\text {total }, u}$ denotes the power constraint of UE $u$. Note that DFT precoding is unitary precoding which preserves the signal energy and it will not change the power distribution of the incoming signal, hence

$$
E\left\{x_{u, n} x_{u, n}^{*}\right\}=\frac{P_{\text {total }, u}}{N} .
$$

The $N \times 2$ outputs of the linear precoder represent two spatial data streams, each of which is processed at one antenna by a $Q$ point IDFT and cyclic prefix is inserted (CP-OFDM). We assume that the assignment of each data stream uses localized subcarrier allocation as applied in LTE for both UEs and they share the same frequency resources. In principle, other allocation methods including IFDMA and B-IFDMA can also be applied. The resulting signal is subsequently parallel to serial converted for transmission. The transmitted signals of both UEs undergo multiple path propagation and are received by the receiver at the $\mathrm{BS}$. The receiver converts the incoming data streams from serial to parallel, removes the cyclic prefix, and processes them using a $Q$ point DFT. Next, the corresponding subcarrier demapping method and ZF-MIMO equalization (EQ) is performed. Subsequently, the equalized signal $\hat{x}_{u, 1}, \ldots, \hat{x}_{\mathrm{u}, N}$ is converted back to the time domain via an $N$ point IDFT for detection. In Figure 1, the block diagram without DFT precoding at the transmitter and IDFT at the receiver is referred to as the inner MIMO OFDMA system.

Our system model only considers single-stream transmission on each subcarrier for each UE. In principle, it is possible for a UE to transmit multiple data streams by applying spatial multiplexing (SM) as discussed in [15] either with or without spatial precoding. However, on one hand, SM for a UE with spatial precoding will generally increase PAPR with respect to single-antenna transmission [15]. On the other hand, the performance of SM for a UE without spatial precoding will be degraded by spatial correlation between the antennas, which is mainly due to the limited antenna separation in the UE. Therefore, it is better to multiplex different data streams from different UEs than from different antennas of the same UE, since the compound virtual MIMO channel benefits from appropriate user grouping and may achieve good rank, even if the two antennas of each UE have high correlation. Moreover, the SC-FDMA transmission structure for each antenna can be preserved in our model hence maintains low PAPR if a common, say frequencyindependent spatial precoder is used for all the subcarriers at a UE. This is especially critical for the cell edge users to be able to bridge the long distance. Furthermore, singlestream transmission is preferred in terms of implementation complexity since coding and transmission can be simply done as in the single-antenna system [16] and hence multiple 


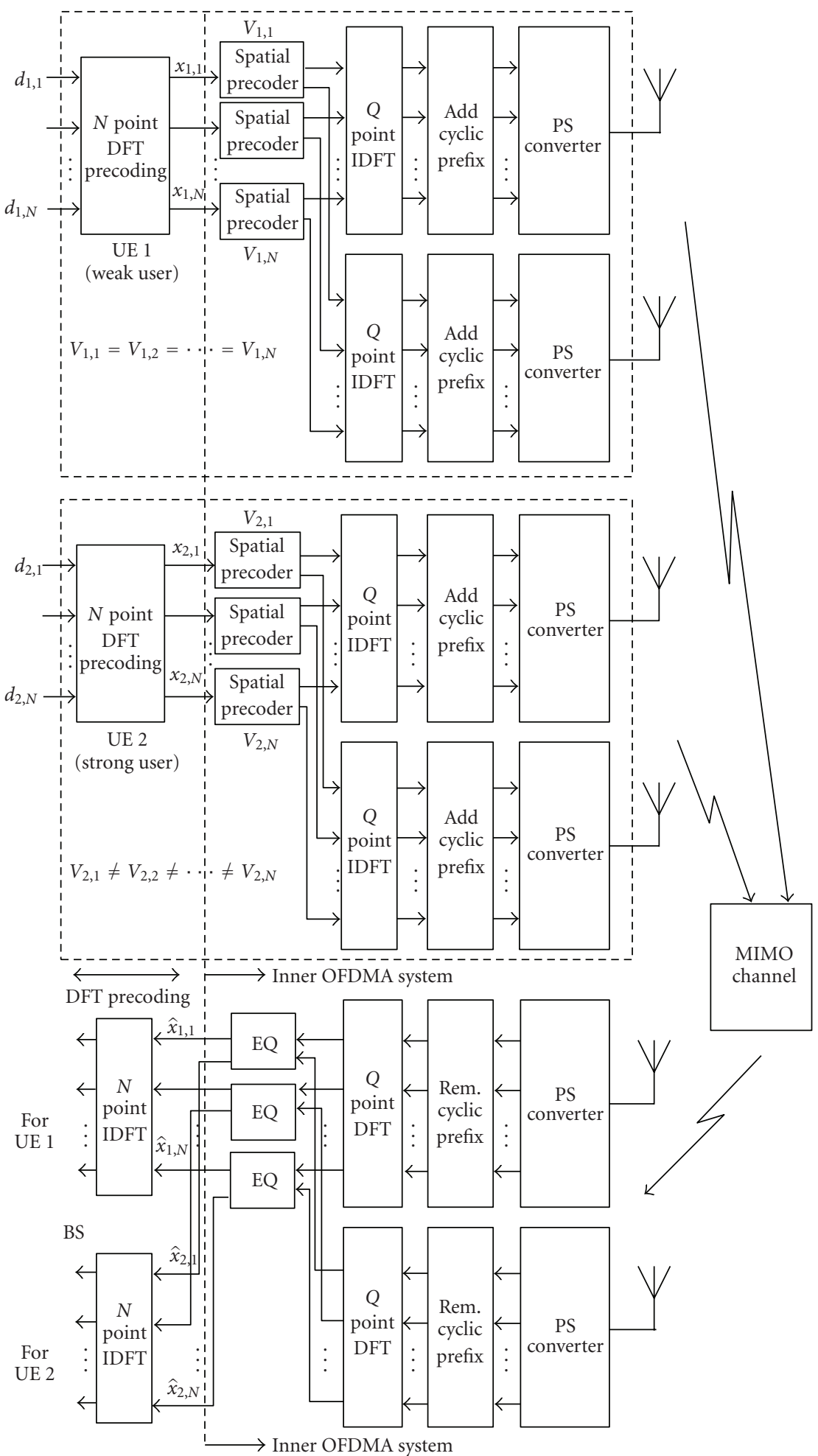

FIGURE 1: Block diagram of the SC-FDMA MIMO system with two UEs under consideration.

antennas can be easily integrated into the conventional single-antenna system.

On each allocated subcarrier, the relationship between the $N$ point DFT output at the transmitter and the $N$ point IDFT input at the receiver can be illustrated as in Figure 2.
Let $\mathbf{G}_{u, n}$ denote the channel matrix between the transmiting antennas of the UE $u$ on subcarrier $n$ and the receiving antennas at the BS (Note that the subcarrier index is counted only in the set of allocated subcarriers). The compound channel of UE $u$ seen by the BS can be obtained by 


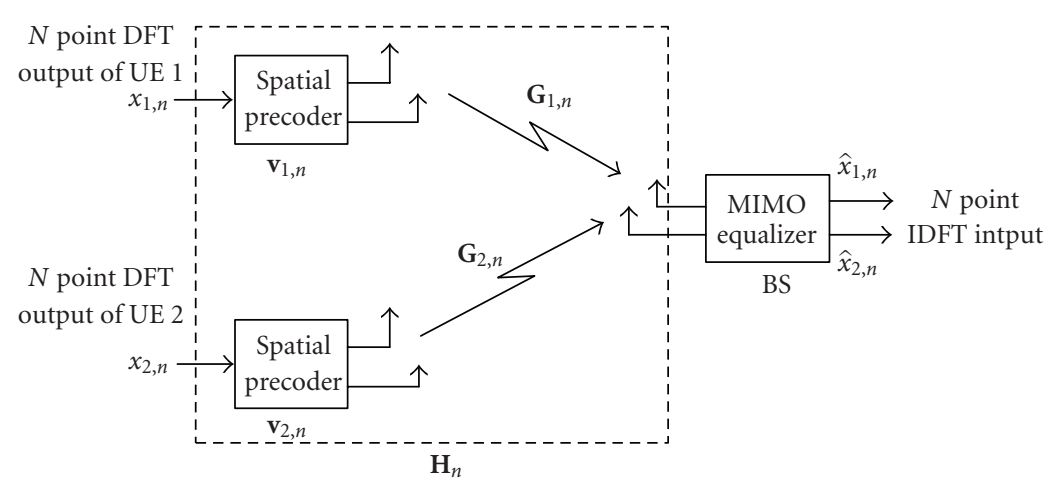

FIGURE 2: Spatial precoder for MIMO uplink with multiple transmit antennas at each UE and multiple antennas at the BS. The index $n$ represents the $n$th allocated subcarrier in the system.

$\mathbf{h}_{u, n}=\mathbf{G}_{u, n} \mathbf{v}_{u, n}$. The multiuser MIMO channel matrix on subcarrier $n$ is written as

$$
\mathbf{H}_{n}=\left[\begin{array}{ll}
\mathbf{h}_{1, n} & \mathbf{h}_{2, n}
\end{array}\right]=\left[\begin{array}{ll}
\mathbf{G}_{1, n} \mathbf{v}_{1, n} & \mathbf{G}_{2, n} \mathbf{v}_{2, n}
\end{array}\right] .
$$

Signals transmitted on subcarrier $n$ from all the UEs are collected in a vector and denoted by $\mathbf{x}_{n}=\left[\begin{array}{ll}x_{1, n} & x_{2, n}\end{array}\right]^{T}$. The received signal on subcarrier $n$ at the BS is then given by $\mathbf{y}_{n}=\mathbf{H}_{n} \mathbf{x}_{n}+\mathbf{n}_{n}$, where $\mathbf{n}_{n}$ is the white Gaussian noise with variance $E\left\{\mathbf{n}_{n} \mathbf{n}_{n}^{H}\right\}=\sigma^{2} \mathbf{I}$. After the linear ZF-MIMO equalization, the postdetection SNR of UE $u$ on subcarrier $n$ in the inner OFDMA system, $\gamma_{u, n}$, can be calculated as

$$
\gamma_{u, n}=\frac{E\left\{\left|x_{u, n}\right|^{2}\right\}}{\sigma^{2}\left[\left(\mathbf{H}_{n}^{H} \mathbf{H}_{n}\right)^{-1}\right]_{u, u}},
$$

where the operator $[\cdot]_{u, u}$ denotes the $u$ th diagonal element of the matrix [ $\cdot]$. The postdetection SNR for the $n$th component at the IDFT outputs for UE $u$ is related with $\gamma_{u, n}$ by [17]

$$
\hat{\gamma}_{u, n}=\frac{N}{\sum_{n=1}^{N}\left(1 / \gamma_{u, n}\right)},
$$

which is the harmonic mean of $\gamma_{u, n}$ and it is the same for all the components. Note that (4) holds regardless of the used subcarrier allocation method. Using Shannon's formula the achievable spectral efficiency of the sub-channel between each input and output in SC-FDMA system for UE $u$ is then given by $\log _{2}\left(1+\hat{\gamma}_{u, n}\right)$ and the system sum rate of the MIMO SC-FDMA system is the rate sum of all the subcarriers of all the UEs [17], that is,

$$
\begin{aligned}
R & =\sum_{u=1}^{2} \sum_{n=1}^{N} \log _{2}\left(1+\hat{\gamma}_{u, n}\right) \\
& =\sum_{u=1}^{2} \sum_{n=1}^{N} \log _{2}\left[1+\frac{N}{\sum_{n=1}^{N}\left(1 / \gamma_{u, n}\right)}\right] .
\end{aligned}
$$

According to (1), (2), (3), and (5), our objective to maximize the system sum rate $R$ can be formulated as follows:

$$
\begin{aligned}
\max _{\mathbf{v}_{1,1}, \ldots, \mathbf{v}_{2, N}} & \sum_{u=1}^{2} N \log _{2}\left(1+\frac{P_{\text {total }, u}}{\sigma^{2} \sum_{n=1}^{N}\left[\left(\mathbf{H}_{n}^{H} \mathbf{H}_{n}\right)^{-1}\right]_{u, u}}\right) \\
\text { s.t. } & \left\|\mathbf{v}_{u, n}\right\|_{2}^{2}=1, \quad u=1,2 ; n=1, \ldots, N,
\end{aligned}
$$

where optimization is performed over all possible precoding vectors subject to the constraints that the precoder is normalized according to the transmit power constraint. Note that our system model also includes the special case that only one antenna is available at each UE (conventional virtual MIMO), by setting $\mathbf{v}_{u, n}$ to $\left[\begin{array}{ll}1 & 0\end{array}\right]^{T}$ or $\left[\begin{array}{ll}0 & 1\end{array}\right]^{T}$ for $u=1,2 ; n=$ $1, \ldots, N$ depending on which antenna is used by the UEs.

\section{Spatial Precoder Optimization}

A direct optimization of the objective function in (6) seems to be very difficult and therefore we look for an approximative solution. According to (5), a higher $\gamma_{u, n}$ for both UEs on subcarrier $n$ in the inner OFDMA system leads to a higher $R$; therefore, to maximize $R$, it is beneficial to maximize $\gamma_{u, n}$, or equivalently the data rate for both UEs in the inner OFDMA system and at the same time taking the objective function (harmonic mean of $\gamma_{u, n}$ 's) into account.

3.1. Eigenbeamforming. If only a single UE, for example, UE $u$ is present and other UEs do not transmit in the system according to Figure 2, the optimum spatial precoder on subcarrier $n$ at the transmitter and equalizer at the receiver is given by the dominant right and left singular vector of $\mathbf{G}_{u, n}$ or equivalently the dominant eigenvector (DEV) of $\mathbf{G}_{u, n}^{H} \mathbf{G}_{u, n}$ and the dominant eigenvector of $\mathbf{G}_{u, n} \mathbf{G}_{u, n}^{H}$, respectively. This transmission strategy is called dominant eigenbeamforming transmission (DET). Under this condition, the postdetection SNR on subcarrier $n$ before IDFT is maximized and this relation can be expressed as

$$
\hat{x}_{u, n}=\sqrt{\lambda_{u, 1}} x_{u, n}+z_{u, n}
$$

where $\lambda_{u, 1}$ is the dominant (largest) eigenvalue of $\mathbf{G}_{u, n}^{H} \mathbf{G}_{u, n}$ for $\mathrm{UE} u$ and $z_{u, n}$ is the AWGN noise with variance $\sigma_{u, n}^{2}$ for UE $u$ 
on subcarrier $n$. Hence the postdetection SNR on subcarrier $n$ can be calculated as

$$
\gamma_{u, n}^{\mathrm{DEV}}=\frac{P_{\text {total }, u}}{\sigma_{u, n}^{2} N} \lambda_{u, 1}
$$

In the case that both UEs are present, if both UEs use DET strategy for transmission, maximum power of both UEs is coupled into the channel but the UEs' signal will generally interfere with each other unless their effective channels happen to be orthogonal to each other, that is, $\mathbf{h}_{1, n}^{H} \mathbf{h}_{2, n}=0$. For this special case, a ZF-MIMO equalizer reduces to a matched filter which maximizes the output SNR of both data streams [18] and thus also maximizes the achievable system sum rate of both UEs on subcarrier $n$.

3.2. Orthogonal Precoder (OP). On the other hand, the transmitted signal from both UEs can always be made interference free to each other if one UE, that is, UE 2, applies a precoding vector in a way such that its effective channel $\mathbf{h}_{2, n}$ is orthogonal to that of the reference UE, that is, UE 1. In other words, the signal of the reference UE will not be disturbed and the system sum rate will increase due to the accommodation of the data stream from the additional UE. For convenience, this precoder is referred to as an orthogonal precoder in the sequel. Denote the effective channel of the reference UE $u$ on subcarrier $n$ as $\mathbf{h}_{u, n}$, the orthogonal precoder $\mathbf{v}_{u^{\prime}, n}$ for the UE $u^{\prime}$ with respect to the reference UE should fulfill

$$
\mathbf{h}_{u, n}^{H}\left(\mathbf{G}_{u^{\prime}, n} \mathbf{v}_{u^{\prime}, n}\right)=0,
$$

where $\mathbf{G}_{u^{\prime}, n}$ is the physical channel of the UE to which an orthogonal precoder should be applied. The solution to (9) can be obtained as

$$
\mathbf{v}_{u^{\prime}, n}^{\perp}=\frac{\mathbf{G}_{u^{\prime}, n}^{-1} \mathbf{h}_{u, n}^{\perp}}{\left\|\mathbf{G}_{u^{\prime}, n}^{-1} \mathbf{h}_{\dot{u}, n}^{\perp}\right\|_{2}^{\perp}},
$$

where $\mathbf{h}_{u, n}^{\perp}$ represents the vector orthogonal to $\mathbf{h}_{u, n}$ and the denominator is used to normalize the power of the precoder.

However, due to the limited degrees of freedom of the linear precoder, after precoding the effective channel orthogonal to the reference UE may experience bad channel condition and therefore the UE which has to transmit the signal in this direction will suffer from low data rate.

In this work, our proposal is to find an appropriate trade-off between completely eliminating the interference (irrespective of how much energy is lost for UE 2) and preserving as much energy as possible for both UEs (at the expense of possibly suffering from interference between the data streams).

\subsection{Combination of DEV Precoder and Orthogonal Precoder.} The fact that the DEV precoder preserves as much energy as possible for both UEs (at the expense of possibly suffering from high interference between the data streams) and the orthogonal precoder completely eliminates the interference (irrespective of how much energy is lost for one of the UEs) suggests that we can find an appropriate trade-off between them. To this end, we propose for each UE a precoder which is the linear combination of its DEV precoder and the orthogonal precoder (with which the resulting beam is orthogonal to the dominant eigenbeam of the other UE), that is,

$$
\mathbf{v}_{u, n}^{\mathrm{DEV}, \perp}=\frac{\alpha_{u, n} \mathbf{v}_{u, n}^{\mathrm{DEV}}+\left(1-\alpha_{u, n}\right) \mathbf{v}_{u, n}^{\perp}}{\left\|\alpha_{u, n} \mathbf{v}_{u, n}^{\mathrm{DEV}}+\left(1-\alpha_{u, n}\right) \mathbf{v}_{u, n}^{\perp}\right\|_{2}},
$$

where the coefficients $\alpha_{u, n}$ and $\left(1-\alpha_{u, n}\right), \alpha_{u, n} \in[0,1]$, define for UE $u$ the weighting for the DEV precoder and the orthogonal precoder, respectively. The denominator of (11) is used to normalize the power of the precoder. Note that for the special case of $\alpha_{u, n}=0$ and $\alpha_{u, n}=1$, the precoder of UE $u$ corresponds to its orthogonal precoder and its DEV precoder, respectively. In order to optimize the system sum rate, the $\alpha_{u, n}$ 's should be optimized jointly over all subcarriers for all UEs.

3.4. Selection Procedure. Using (11) as the spatial precoder for each UE, the problem of maximizing the system sum rate in the ZF-equalized MIMO SC-FDMA system with two UEs can be reformulated as finding an optimum $\alpha_{u, n}$ for the linear combination of its DEV and its orthogonal precoder such that the system sum rate is maximized. Consequently, (6) can be rewritten as

$$
\begin{aligned}
\max & \sum_{u=1}^{2} N \log _{2}\left(1+\frac{P_{\text {total }, u}}{\sigma^{2} \sum_{n=1}^{N}\left[\left(\mathbf{H}_{n}^{H}\left(\boldsymbol{\alpha}_{n}\right) \mathbf{H}_{n}\left(\boldsymbol{\alpha}_{n}\right)\right)^{-1}\right]_{u, u}}\right) \\
\text { s.t. } & 0 \leq \alpha_{1, n} \leq 1, \quad 0 \leq \alpha_{2, n} \leq 1, \quad n=1, \ldots, N,
\end{aligned}
$$

where

$$
\begin{gathered}
\boldsymbol{\alpha}_{n}=\left[\alpha_{1, n}, \alpha_{2, n}\right] \\
\mathbf{H}_{n}=\left[\begin{array}{c}
\left(\mathbf{G}_{1, n}\left[\alpha_{1, n} \mathbf{v}_{1, n}^{\mathrm{DEV}}+\left(1-\alpha_{1, n}\right) \mathbf{v}_{1, n}^{\perp}\right]\right)^{T} \\
\left(\mathbf{G}_{2, n}\left[\alpha_{2, n} \mathbf{v}_{2, n}^{\mathrm{DEV}}+\left(1-\alpha_{2, n}\right) \mathbf{v}_{2, n}^{\perp}\right]\right)^{T}
\end{array}\right]^{T}
\end{gathered}
$$

is the compound channel matrix on subcarrier $n$ in the system. In the above optimization problem, the weighting factors $\alpha_{u, n}$ have to be optimized jointly among all users and all subcarriers. There are mainly two issues associated with it. The first issue is related to the PAPR of the transmit waveform. Due to the frequency selectivity of the channels, the optimal precoding vector will vary from subcarrier to subcarrier in general. Such frequency-dependent precoding vectors, if applied, will destroy the single carrier structure of the transmitted signal. Note that applying precoding vectors after DFT in the frequency domain is equivalent to a convolution and summation of the data symbols in the time domain [15], thus PAPR of the composite transmitted signal will increase with respect to single antenna transmission. The other issue is related with computational complexity, which increases exponentially in the number of subcarriers $N$ and 
in the number of UEs $U$. We will address these two issues separately in the following.

To address the PAPR issue, we propose to use a frequency-independent spatial precoder for one UE, preferably the weaker UE. As a result, the single carrier structure of the transmit signal and hence the low PAPR property at each antenna for this UE can be maintained. In this work, we use the dominant eigenvector of the average correlation matrix $(1 / N) \sum_{n=1}^{N}\left(\mathbf{G}_{n}^{H} \mathbf{G}_{n}\right)$ as the precoding vector, where the subscription $u$ is dropped here for notational simplicity. In addition, since the same precoder is applied for all subcarriers, the signal processing complexity is reduced and the signalling overhead to inform the UE about the precoder is also reduced considerably. Subsequently, the best coefficient of $\alpha$ is found numerically for the other UE such that the system sum rate is maximized and its resulting precoding vector is referred to as the optimum complementary precoder (OCP) in our context.

In order to reduce the computational complexity of the selection procedure, optimization of (12) can be performed on an arbitrary subcarrier first to obtain the best precoder for that subcarrier and then it is considered fixed for the optimization of the next subcarrier. As a result, the computational complexity is linear in the number of subcarriers. A description of the algorithm with two UEs can be found in Algorithm 1.

Algorithm 1 aims to maximize the rate sum of all UEs. It can also be extended to incorporate different weighting for the rate of different UEs so as to maximize the weighted sum rate of all UEs. By introduction of the weighting factor $w_{u}$ for the rate $R_{u}$ of the $u$ th $\mathrm{UE}$, the two user weighted sum rate problem is $R_{\text {total }}=\sum_{u=1}^{2} w_{u} R_{u}$ and the optimal $\alpha_{2, n \text {,opt }}$ in Algorithm 1 should be modified as

$$
\begin{aligned}
& \alpha_{2, n, \mathrm{opt}} \\
& =\operatorname{argmax} \sum_{u=1}^{2} w_{u} N \cdot \log _{2}\left(1+\frac{P_{\text {total }, u} / \sigma^{2}}{k_{u}+\left[\left(\mathbf{H}_{n}^{H}\left(\alpha_{2, n}\right) \mathbf{H}_{n}\left(\alpha_{2, n}\right)\right)^{-1}\right]_{u, u}}\right) .
\end{aligned}
$$

This modified version of Algorithm 1 dealing with the weighted sum rate problem is related to the achievable rate region in the system, which will be interesting for resource allocation and QoS optimization. Changing the weights, any point on the boundary of the achievable rate region can be achieved.

3.5. Scheduling. In Algorithm 1, one UE, for example, UE 1, always utilizes its dominant eigenbeam direction and then UE 2 has to transmit in a direction such that the system sum rate is maximized. The resulting transmission direction of UE 2 generally differs from its own dominant eigenbeam direction. It can be expected that in the case of both UEs having similar channel conditions, on average the postdetection SNRs of UE 1 in the inner OFDMA system are higher than those of UE 2. According to (4), higher postdetection SNRs lead to a higher harmonic mean, corresponding to the postdetection SNRs of the SC-FDMA system. Therefore,

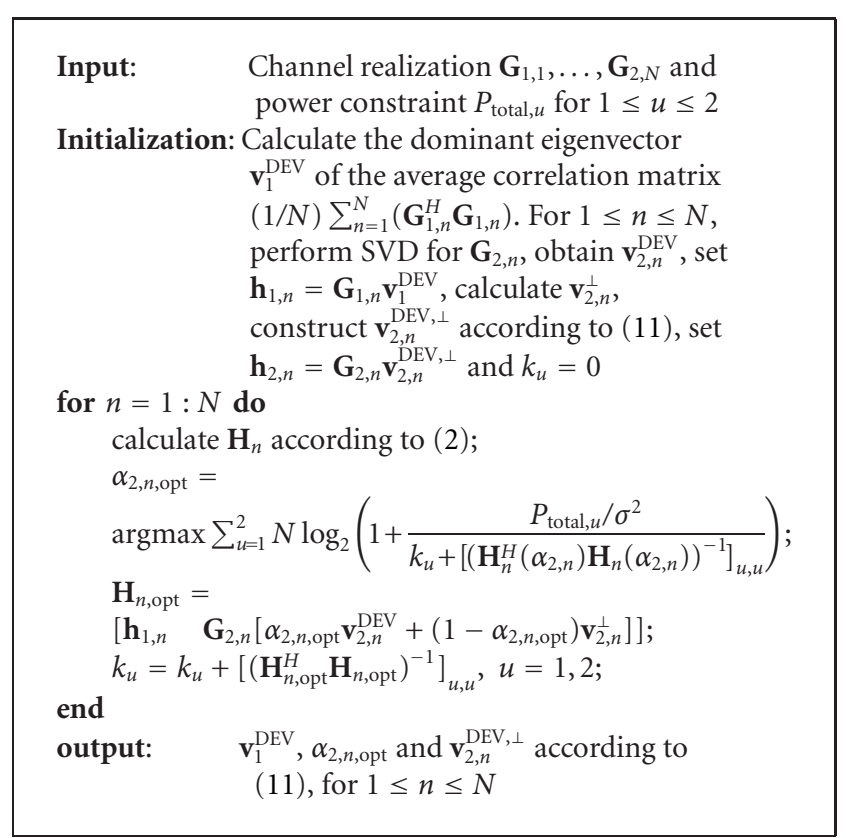

Algorithm 1: Spatial precoder optimization algorithm.

it follows that on average the rate of UE 1 is higher than that of UE 2 in the MU-MIMO SC-FDMA system. From the UEs' perspective, this fixed optimization order is biased and it may cause the individual rate of the UEs to differ a lot from each other. Another factor which may affect the individual rate of the UEs is that the UE which uses OCP may produce strong interference to the UE which uses the DEV precoder. This will not cause any problem if both UEs have similar channel conditions. However, if their channel qualities are largely unbalanced (e.g., $10 \mathrm{~dB}$ difference), even the weaker UE always transmits in its dominant eigenbeam direction, a small amount of interference from the much stronger UE will have a strong impact on the rate of the weaker UE. In this situation, it is desirable to let the stronger UE transmit in the direction orthogonal to that of the weaker UE. This leads to our following simple scheduling algorithm to mitigate the aforementioned problems and to balance the individual rate of the UEs.

The scheduler works as follows. It keeps track of the average rate $R_{\mathrm{avg}, u}$ of each $\mathrm{UE}$, which will be updated on per subframe basis. In subframe $t$, the scheduling algorithm assigns the DEV precoder to UE $u^{*}$ with smaller $R_{\mathrm{avg}, u}$ in the system, which aims to give higher priority to the weaker UE to balance the individual user rate. In addition, in order to avoid interfering the rate of weaker UE if the UEs experience largely unbalanced channel conditions in the system, a weighting factor $\beta$ is introduced to weight the channel orthogonal to that of the weaker UE by setting

$$
h_{\tilde{u}, n}= \begin{cases}\beta \mathbf{G}_{\tilde{u}, n} \frac{\mathbf{v}_{\tilde{u}, n}^{\perp}}{\left\|\mathbf{v}_{\tilde{u}, n}^{\perp}\right\|_{2}}, \quad \alpha=0, \beta \geq 1, \\ \mathbf{G}_{\tilde{u}, n} \mathbf{v}_{\tilde{\mathrm{DEV}}, n}, \quad \alpha \neq 0\end{cases}
$$


in the precoder optimization algorithm, where $\tilde{u}$ denotes the UE with higher average rate $R_{\text {avg }, u}$. In (15), choosing a larger $\beta$ means to virtually boost the quality of the channel orthogonal to the transmission direction of the weaker UE, so that it is treated as a good channel and the selection procedure preferably picks it up for the stronger UE. In other words, a bigger $\beta$ indicates higher importance that the UE with higher average rate in the past should transmit in a direction which does not cause any interference to the UE with lower average rate in the past and vice versa.

\section{Simulation Results}

To evaluate the performance of the proposed spatial precoders in a $2 \times 2$ uplink MIMO system with two UEs as shown in Figure 2, simulations are conducted in the 3GPP LTE uplink with the parameter assumptions given in Table 1 .

A snapshot of the subcarrier channel power gain between the UEs and the BS is illustrated in Figure 3. For simplicity, it is further assumed that each resource block (RB) experiences the same channel condition and its channel frequency response is represented by the middle, that is, the 6th, subcarrier of the RB. Under this condition, performance evaluation can be conducted per RB basis and the concept meant for a subcarrier in our previous discussion can be directly applied to an $\mathrm{RB}$ to reduce the computational complexity. In the following, first the performance is evaluated using a channel snapshot for illustrative purpose. Then we present results in terms of average spectral efficiency for different bandwidth and SNR conditions.

The upper part of Figure 4 shows the postdetection SNR $\gamma_{u, n}$ of the inner OFDMA system using a channel snapshot of $20 \mathrm{MHz}$ (totally $100 \mathrm{RBs}$ ) as depicted in Figure 3. Note that only $20 \mathrm{RBs}$ (240 subcarriers) are shown for better visibility of the details. The dashed lines represent the results for the conventional UEs with each having only a single antenna, that is, setting $\mathbf{v}_{u, n}=\left[\begin{array}{ll}1 & 0\end{array}\right]$ for all $u$ 's and $n$ 's in our model. The solid lines stand for the results obtained by the proposed precoding scheme where UE 1 employs the dominant EV of the average channel correlation matrix as precoders for all the subcarriers and UE 2 uses the OCP for each subcarrier. The spectral efficiency of each sub-channel between each input and output component in the SC-FDMA system is plotted in the lower part of Figure 4. It can be observed that the proposed algorithm significantly improves the spectral efficiency, or in other words the rate of the UEs in the system.

Next, the statistics of the postdetection SNR is studied for the conventional virtual MIMO SC-FDMA and the proposed MIMO SC-FDMA with spatial precoding, where a setting of two UEs with the same average SNR $=10 \mathrm{~dB}$ over 15 RBs $(\approx 3 \mathrm{MHz})$ is assumed. Statistics are collected from 50 randomly distributed locations in a cell and 100 subframes are considered assuming that each UE randomly moves from each location. The complementary cumulative distribution function (CCDF) of the postdetection SNR for both schemes is compared in Figure 5, which shows the probability that the postdetection SNR is larger than a certain value. It can be seen that with the proposed spatial precoding scheme the postdetection SNRs of both UEs are significantly increased,
TABLE 1: Parameter assumptions for simulation.

\begin{tabular}{lc}
\hline Parameters & Assumption \\
\hline Carrier frequency & $2 \mathrm{GHz}$ \\
Transmission bandwidth & $3 \mathrm{MHz}, 20 \mathrm{MHz}$ \\
Subframe duration & $1 \mathrm{~ms}$ \\
Subcarrier spacing & $15 \mathrm{KHz}$ \\
Number of subcarriers & 180,1200 \\
Number of subcarriers per RB & 12 \\
Channel model & 3GPP SCME urban macro [19] \\
Number of UEs & 2 \\
Number of BSs & 1 \\
Antennas per UE & 2 \\
Antennas per BS & 2 \\
UE antenna spacing & 0.5 wavelength \\
BS antenna spacing & 10 wavelengths \\
UE velocity & $10 \mathrm{~m} / \mathrm{s}$ \\
\hline
\end{tabular}

where the improvement for the UE using dominant EV precoding is much larger than that for the UE using OCP.

For the same setting, Figure 6 shows the cumulative distribution function (CDF) of the system spectral efficiency and the individual user spectral efficiency for the conventional virtual MIMO SC-FDMA and the proposed MIMO SC-FDMA with spatial precoding. Spectral efficiency for UE 1 and UE 2 is indicated by dashed and dashdot curves, respectively. The system spectral efficiency is indicated by the solid curve. It can be observed that in comparison with the conventional virtual MIMO SC-FDMA the 50 percentile achievable system spectral efficiency almost doubles after applying the proposed scheme for different fixed optimization orders. It can also be clearly seen that with the fixed optimization orders, the UE using the dominant EV precoder always has higher average spectral efficiency than the one using OCP. If the proposed spatial scheduler is used, individual spectral efficiency of the UEs are balanced and the CDF of the achievable system spectral efficiency (green) lies almost on top of those obtained by fixed optimization orders (red and blue). For reference, the case that both UEs use frequency-dependent precoding according to (11) is also shown for both the SC-FDMA system and the OFDMA system. Note that although higher spectral efficiency is possible in this case, the single-carrier structure and hence the low PAPR property cannot be maintained for both UEs any more.

Figure 7 depicts the performance comparison assuming $100 \mathrm{RBs}$ are available in the system and other parameters remain the same as in the previous simulation. It can be seen that both the conventional virtual MIMO SC-FDMA and the proposed precoding schemes have inferior performance to the case with $15 \mathrm{RBs}$ (cf. Figure 6). The reason is as follows. In the system with relatively small bandwidth, diversity offered in the frequency domain is limited hence all subcarriers within the system bandwidth experience similar channel conditions, that is, in some subframes channels are good and in others they are bad. As the transmission bandwidth increases, diversity offered in the bandwidth 


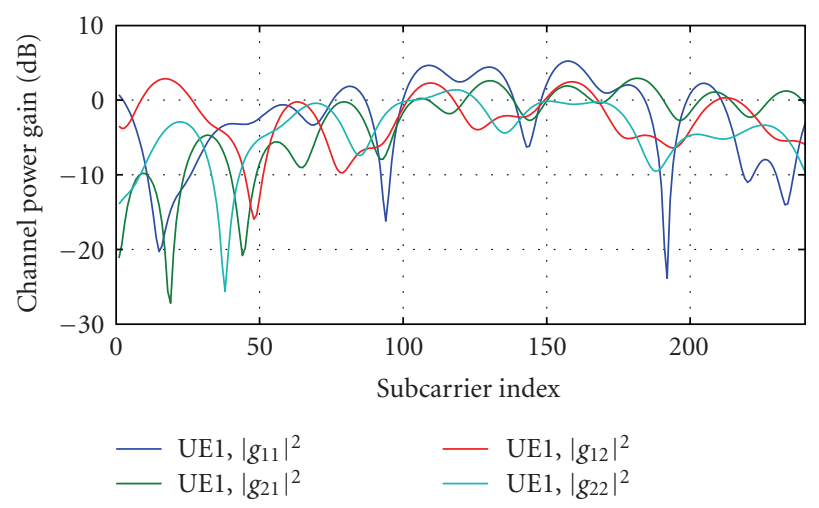

(a)

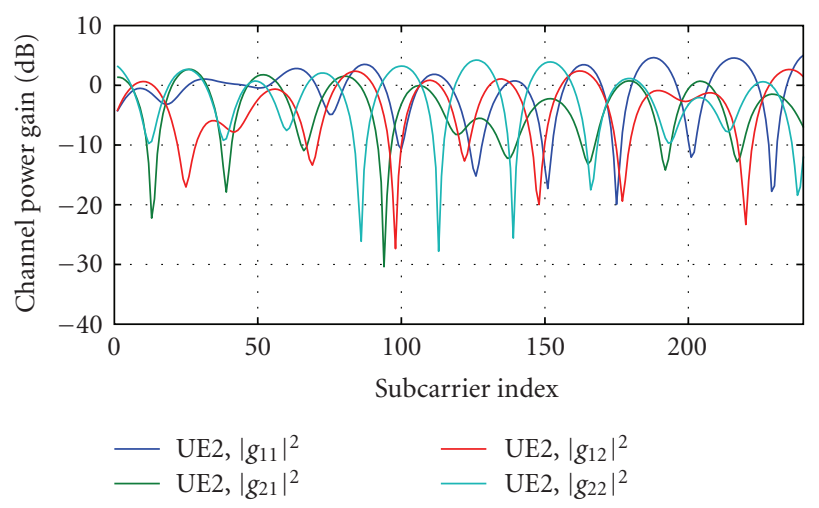

(b)

FIGURE 3: Channel power gain snapshot between the UEs and the BS according to Figure $2 ; g_{i j}$ denotes the subcarrier frequency response between the $j$ th transmitter of the UE and the $i$ th receiver at the BS.

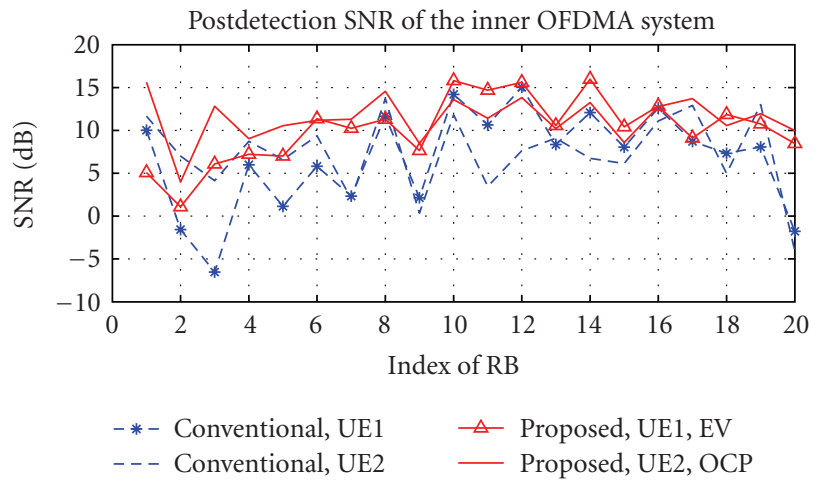

(a)

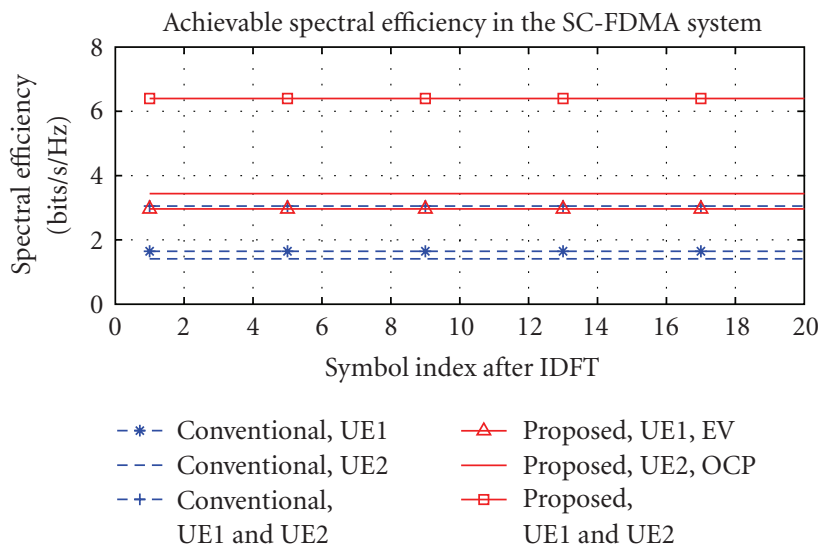

(b)

FIGURE 4: Performance comparison between the conventional virtual MIMO SC-FDMA and the proposed MIMO SC-FDMA with precoding using the channel snapshot in Figure 3. (Upper: postdetection SNR of the inner OFDMA system; Lower: achievable spectral efficiency in the SC-FDMA system.)

is higher. As a result, all subframes consist of a similar number of strong/weak subcarriers and a similar number of subcarriers in which both UEs have similar spatial signatures. It follows that the number of low postdetection SNRs in the inner OFDMA system is similar in all subframes. Since the postdetection SNRs of all components in the SC-FDMA system are the harmony mean of the postdetection SNRs of the inner OFDMA system and they are mainly restricted by small values, it turns out that having similar number of low postdetection SNRs for each subframe is less spectrally efficient than having more low postdetection SNRs for some subframes and less for the others [20]. Nevertheless, the proposed scheme still achieves about twice as high system spectral efficiency as the conventional scheme.

Figure 8 compares the performance with unequal average received SNR for different UEs, that is, $\mathrm{SNR}_{\mathrm{UE} 1}=0 \mathrm{~dB}$ and $\mathrm{SNR}_{\text {UE2 }}=10 \mathrm{~dB}$ in the $3 \mathrm{MHz}$ bandwidth. About twice the system spectral efficiency with respect to the conventional scheme can be expected from the proposed spatial precoding schemes for different fixed optimization orders. It can be seen that the scheme which always gives higher priority to the weaker UE, that is, DEV and OCP are applied to the weaker UE and the stronger UE, respectively, still results in significant lower rate for weaker UE (red dashed) than for the stronger UE (red dashdot). This is due to the strong amount of interference caused by the stronger UE. Nevertheless, by applying our prosed scheduling algorithm (green) with $\beta=$ 10, comparable individual rate of the UEs can be achieved.

Figures 9 and 10 illustrate the achievable average rate (bits/s/Hz) obtained by using the modified version of Algorithm 1 (which incorporates weighted sum rate maximization, cf. (14)) in a two-UE SCME urban macro scenario according to Table 1 for different SNR conditions. Only the case with $3 \mathrm{MHz}$ system bandwidth is considered and the rate results are evaluated over 2000 subframes. The boundary point is computed with the modified Algorithm 1 for 33 different weights with $w_{1}$ ranging from 0.01 to 1.99 in steps of $0.06\left(w_{2}=2-w_{1}\right)$. The red curve is obtained by choosing the $\mathrm{DEV}$ of the average channel correlation matrix as the spatial precoder for UE 1 and choosing OCP for each subcarrier 


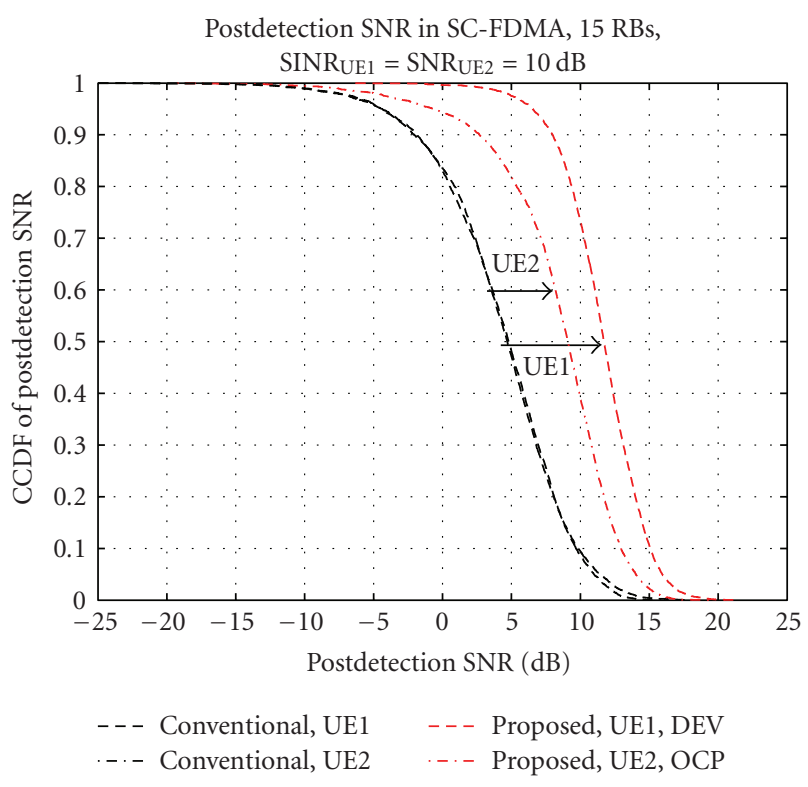

FIgURE 5: Complementary cumulative distribution function (CCDF) of the postdetection SNR between the conventional MIMO SC-FDMA and the proposed MIMO SC-FDMA with spatial precoding. $15 \mathrm{RBs}$ are available in the system and both UEs have the same average received SNR of $10 \mathrm{~dB}$.

for UE 2 (by optimizing the linear combination of DEV and orthogonal precoder) such that the weighted sum rate of two UEs is maximized. The blue curve is obtained by the opposite optimization order, that is, choosing DEV for UE 2 and OCP for UE 1 . If the UEs have the ability to coordinate the timing, the rate pairs on the black dashed curve (but not on the blue and red curves) can be achieved by time-sharing.

The UE rate pairs at the two ends of the black dashed curve correspond to the case where strongly different weighting factors are applied to different UEs ( $w=0.01$ for one UE and $w=1.99$ for the other). They also correspond to case where the UE with higher weighting using DEV precoder and the UE with lower weighting using the orthogonal precoder (OP). Imposing higher weighting to the UE means giving higher priority to the UE to maximize its own data rate, then the UE with lower weighting has to transmit in the direction without causing strong interference to the UE with higher weighting. The extreme case is that the UE with lower weighting chooses the OP such that it does not cause any interference to the UE with higher weighting.

For comparison, three additional transmit-precoding strategies are also considered and their achievable rate performances are shown in the figures. Each strategy applies a frequency-independent precoder on all subcarriers and the precoder can be different for different subframes. The first strategy is that each UE uses its own DEV of the average channel correlation matrix as the spatial precoder. In other words, each UE roughly couples maximum power into the channel without taking care of how much interference will be caused to each other. The second strategy is that each UE adaptively selects one precoder from the 4 predefined

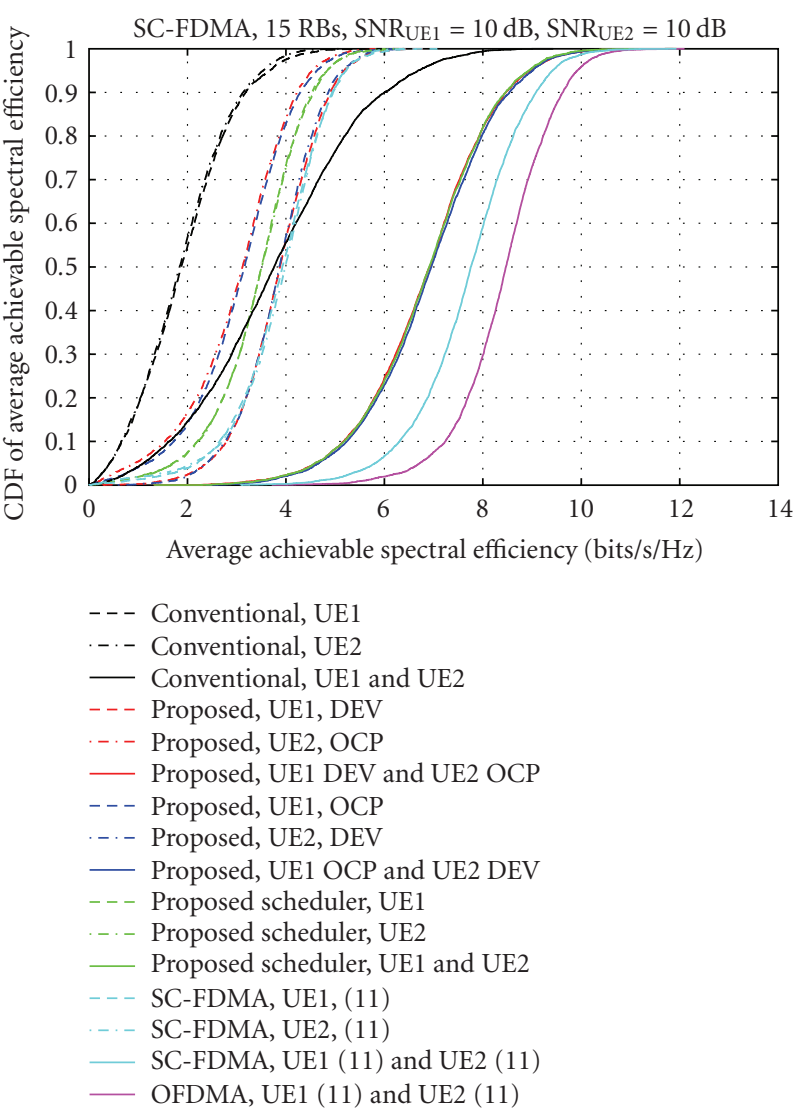

Figure 6: Cumulative distribution function (CDF) of the achievable spectral efficiency by using conventional virtual MIMO with a single antenna per UE (black) and by using a spatial precoder according to Figure 2 with fixed optimization order (red and blue) and a spatial scheduler (green) for an SC-FDMA system $(\beta=1)$. For reference, the case that both UEs use frequency-dependent precoding according to (11) is included for the SC-FDMA system (cyan) and for the OFDMA system (magenta). $15 \mathrm{RBs}$ are available in the system and both UEs have the same average received SNR of $10 \mathrm{~dB}$.

precoding vectors (They are the $\mathbf{v}_{1}=(1 / \sqrt{2})\left[\begin{array}{ll}1 & 1\end{array}\right]^{T}, \mathbf{v}_{2}=$ $(1 / \sqrt{2})[1-1]^{T}, \mathbf{v}_{3}=(1 / \sqrt{2})[1 \quad j]^{T}$, and $\mathbf{v}_{4}=(1 / \sqrt{2})[1 \quad-$ $j]^{T}$, resp.) specified in LTE systems [21] (referred to as the LTE DFT codebook) such that the system sum rate is maximized (16 combinations). The last strategy is that each UE adaptively selects one antenna for transmission (totally 4 possibilities) such that the system sum rate is maximized. It can be observed that all these transmit precoding strategies have inferior rate performance to the proposed scheme. Especially at low and moderate SNR conditions, the gain achieved by the proposed scheme is shown up to $40 \%$ over the adaptive LTE DFT codebook strategy and up to $70 \%$ over the other considered strategies.

For unequal SNR conditions of the UEs, the rate achieved by all the transmit precoding schemes exhibits bias toward the stronger UE. Obviously as shown in Figure 10, the proposed precoding scheme outperforms other considered schemes. 


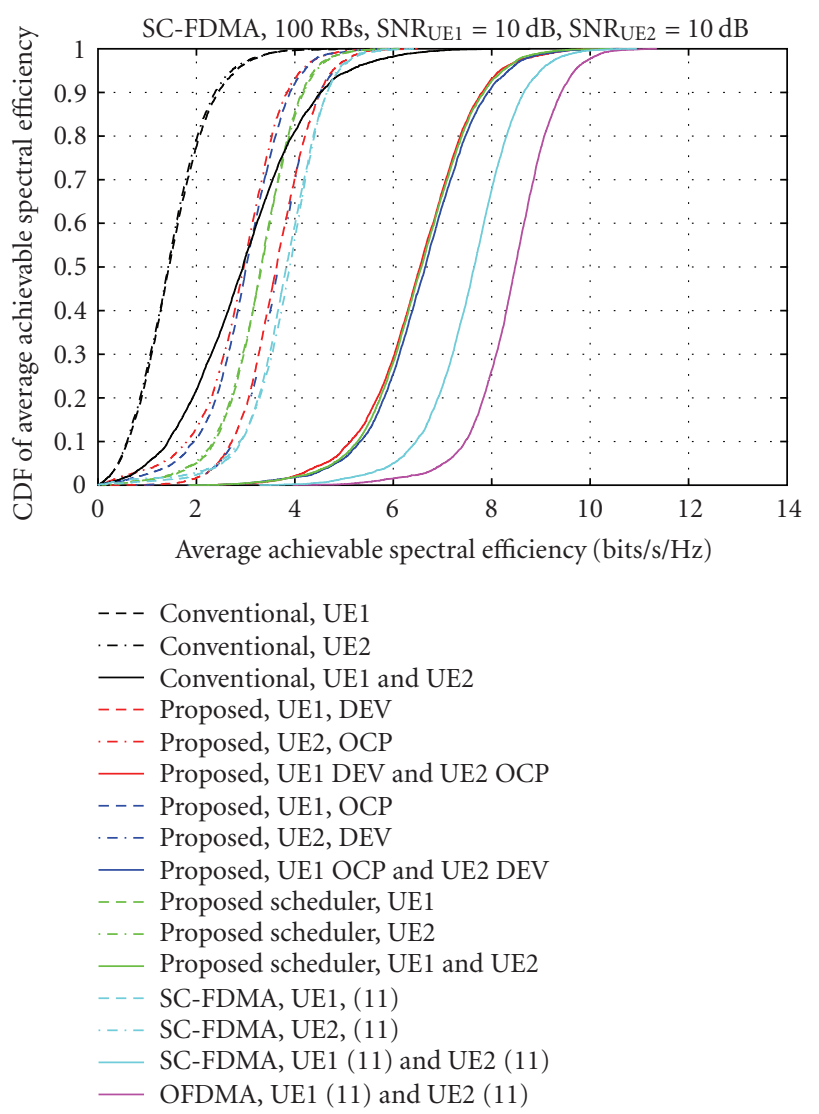

Figure 7: Parameter setting is the same with Figure 6 but 100 RBs are available in the system.

\section{Conclusions}

We propose to apply DFT precoding combined with spatial precoding to optimize the system sum rate in a MIMO SCFDMA uplink. Depending on the requirements, at least one of the UEs can optionally apply a frequency nonselective precoding to obtain a single-carrier waveform with low PAPR. The required feedback overhead to convey the precoder decision to the UE is significantly reduced. Furthermore, to handle the fairness issues between the UEs, a simple spatial scheduler has been proposed within the framework to effectively steer and balance the individual user rate exemplarily. Simulation results show that the system spectral efficiency almost doubles for various SNR conditions compared to the case where each UE has only one transmit antenna. Finally, weighted sum rate maximization is also incorporated in the algorithm and its achievable rate region is presented and potential gains are shown compared to other popular precoding schemes for LTE uplink scenarios.

\section{Open Problems and Future Work}

For the scenario with more than two UEs and more than two antennas in the system, the principle of Algorithm 1 can be extended by constructing candidate precoders $\mathbf{v}_{u, n}^{\mathrm{DEV}, \perp}$ for UE

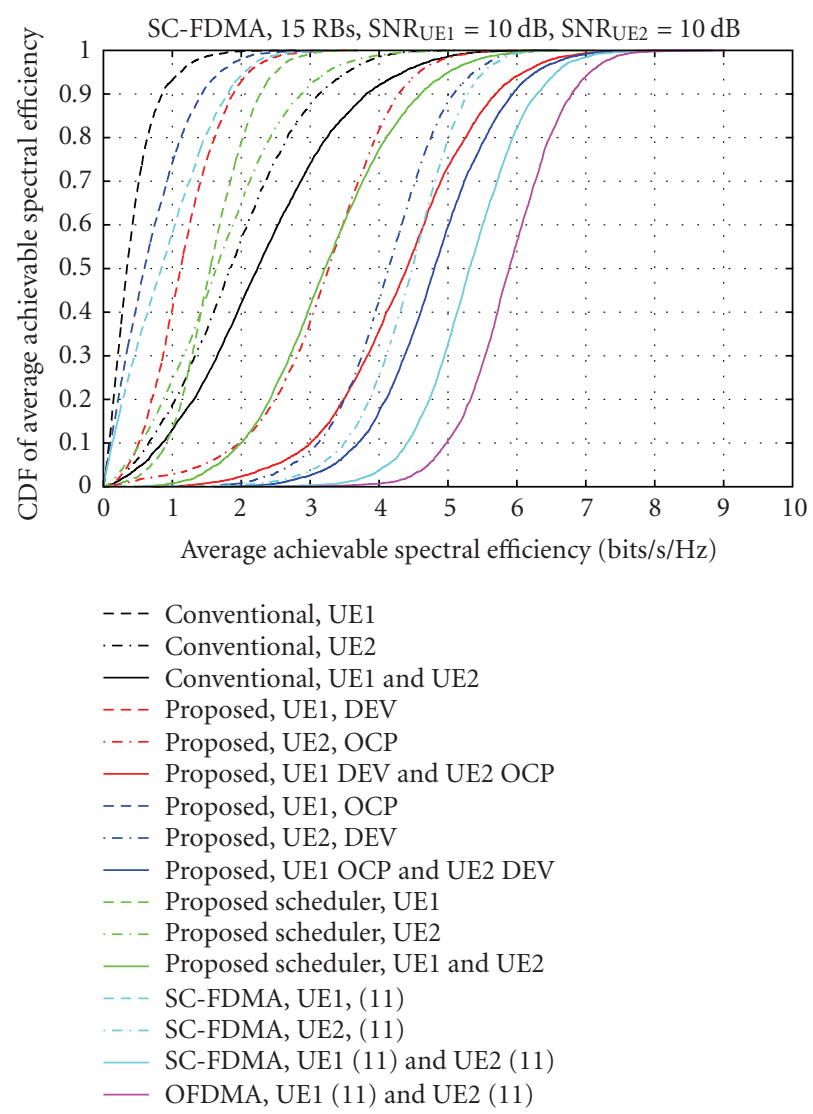

Figure 8: Parameter setting is the same with Figure 6 but with unequal average received SNR for the UEs: $\mathrm{SNR}_{\mathrm{UE} 1}=0 \mathrm{~dB}$ and $\mathrm{SNR}_{\mathrm{UE} 2}=10 \mathrm{~dB} ; \beta=10$.

$u(2 \leq u \leq U)$ according to (11), where $\mathbf{v}_{u, n}^{\mathrm{DEV}}$ can be calculated by performing singular value decomposition (SVD) for the associated channel matrix and $\mathbf{v}_{u, n}^{\perp}$ can be obtained by using (10). However, it might happen that the solution to (10) does not exist. Therefore, advanced algorithm design is needed. A partial solution to accommodate multiple UEs is to divide the whole available system bandwidth into small partitions and apply the proposed algorithm for each partition with two UEs.

In the proposed scheduling algorithm, the introduction of a weighting factor $\beta$ is shown to be able to balance the individual user rates to some extent. A typical value of $\beta$ is between 1 and 10 depending on the channel quality of different users. However, the optimization of $\beta$ for different channel conditions to achieve comparable rates for all users is an open question and subject to future study.

The proposed algorithm assumes that perfect channel state information is available at the BS which only provides an upper bound for the system performance. In a practical system, channel state information estimated in the BS will not be perfect. Nevertheless, the proposed algorithm can be beneficial to improve the system sum rate in LTE femtocell (home BS) [22] scenarios, where UEs typically move at very 


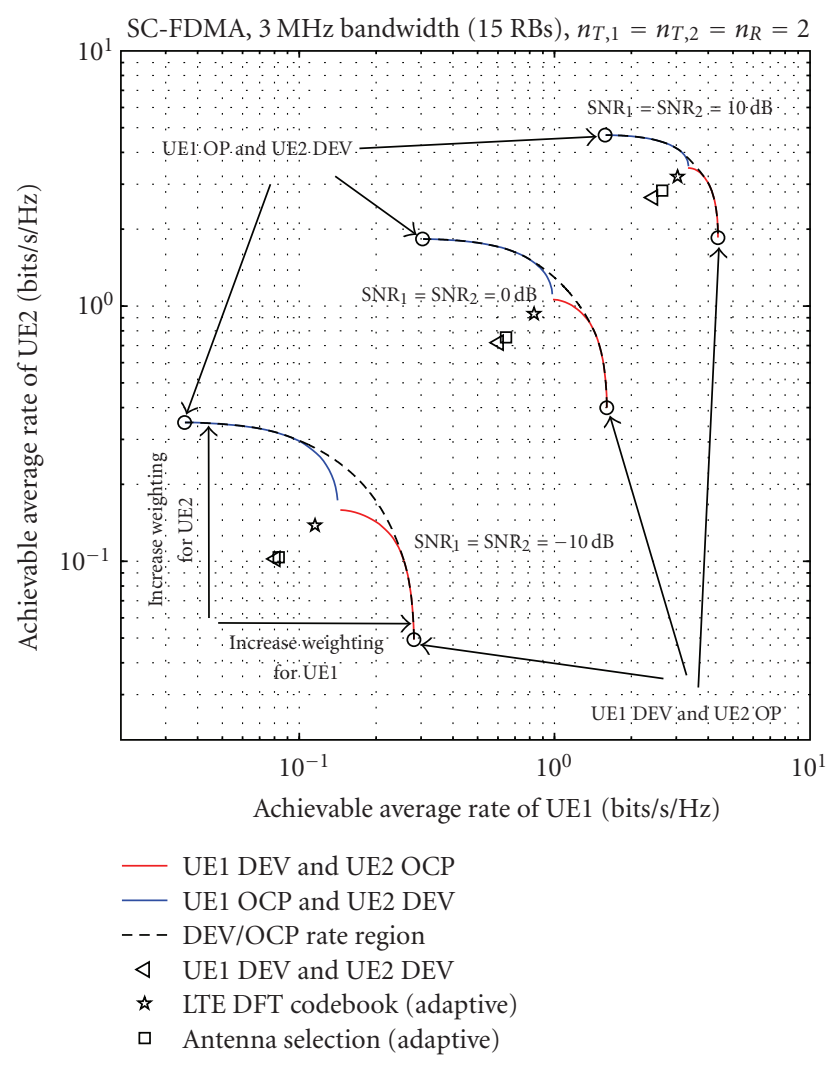

FIGURE 9: Achievable average rate for two UEs for different transmit precoding strategies in the SCME "urban macro" scenario according to Table 1. Both UEs share $3 \mathrm{MHz}$ bandwidth to communicate with the serving BS using the same time and frequency resources. Both UEs have the same SNR.

low speed in a limited area. In such scenarios, channels of all UEs are quasic-static, which makes it possible for the BS to continuously improve channel estimation with the help of the reference signals [21] sent from the UEs.

\section{References}

[1] J. T. E. McDonnell and T. A. Wilkinson, "Comparison of computational complexity of adaptive equalization and OFDM for indoor wireless networks," in Proceedings of the 7th IEEE International Symposium on Personal, Indoor and Mobile Radio Communications (PIMRC'96), pp. 1088-1090, October 1996.

[2] S. H. Han and J. H. Lee, "An overview of peak-to-average power ratio reduction techniques for multicarrier transmission," IEEE Wireless Communications, vol. 12, no. 2, pp. 56-65, 2005.

[3] H. Wu and T. Haustein, "Energy and spectrum efficient transmission modes for the 3GPP-LTE uplink," in Proceedings of IEEE International Symposium on Personal, Indoor and Mobile Radio Communications (PIMRC '07), Athens, Greece, September 2007.

[4] H. G. Myung, J. Lim, and D. J. Goodman, "Peak-to-average power ratio of single carrier FDMA signals with pulse shaping," in Proceedings of the 17th IEEE International Symposium on Personal, Indoor and Mobile Radio Communications (PIMRC '06), Helsinki, Finland, September 2006.

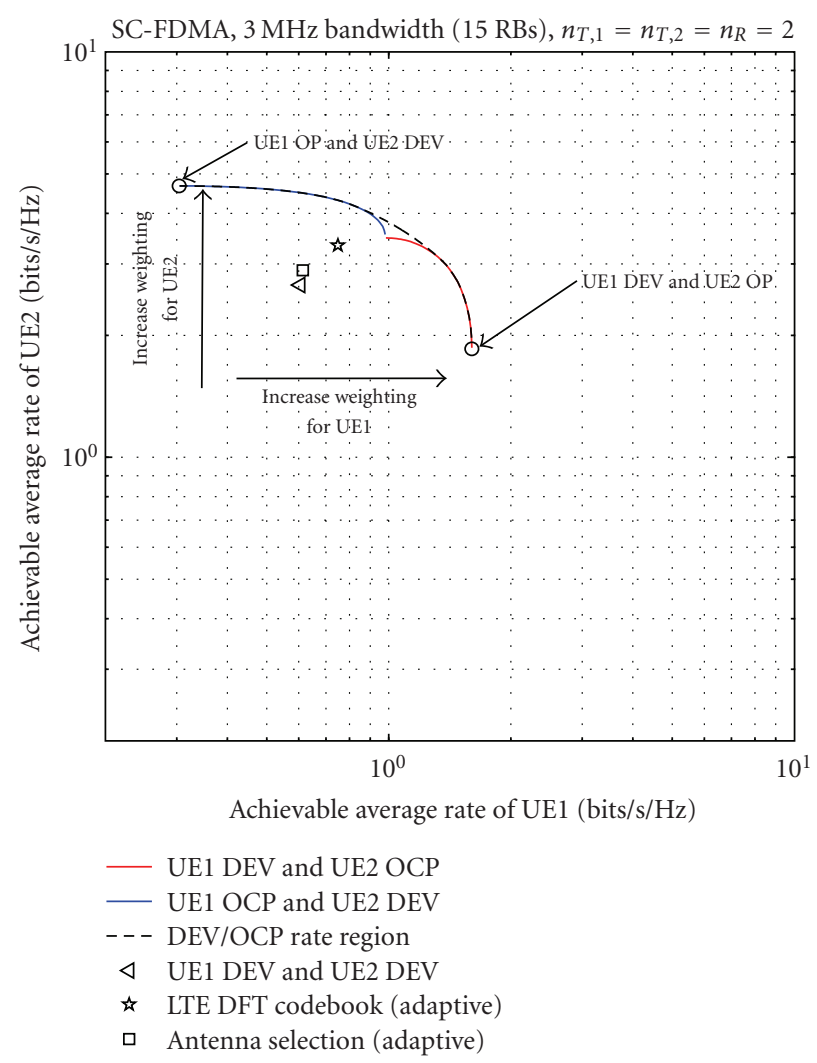

FIgURE 10: Achievable average rate for two UEs for different transmit precoding strategies in the SCME "urban macro" scenario according to Table 1 . Both UEs share $3 \mathrm{MHz}$ bandwidth to communicate with the serving BS using the same time and frequency resources. Unequal SNR with $10 \mathrm{~dB}$ difference is assumed for the UEs $\left(\mathrm{SNR}_{\mathrm{UE} 1}=0 \mathrm{~dB}, \mathrm{SNR}_{\mathrm{UE} 2}=10 \mathrm{~dB}\right)$.

[5] V. Jungnickel, T. Hindelang, T. Haustein, and W. Zirwas, "SCFDMA waveform design, performance, power dynamics and evoluation to MIMO," in Proceedings of IEEE International Conference on Portable Information Devices, Orlando,Fla, USA, March 2007.

[6] H. G. Myung, "Introduction to single carrier FDMA," in Proceedings of the 15th European Signal Processing Conference (EUSIPCO '07), Poznan, Poland, September 2007.

[7] 3GPP TSG RAN, "Some aspects of single-carrier transmission for EUTRA,” R1-050765, August-September 2005.

[8] U. Sorger, I. De Broeck, and M. Schnell, "Interleaved FDMAa new spread-spectrum multiple-access scheme," in Proceedings of IEEE International Conference on Communications (ICC'98), vol. 2, pp. 1013-1017, Atlanta, Ga, USA, June 1998.

[9] T. Svensson, T. Frank, D. Falconer, M. Sternad, E. Costa, and A. Klein, "B-IFDMA-a power efficient multiple access scheme for non-frequencyadaptive transmission," in IST Mobile and Wireless Communications Summit, Budapest, Hungary, July 2007.

[10] T. Frank, A. Klein, and T. Haustein, "A survey on the envelope fluctuations of DFT precoded OFDMA signals," in Proceedings of IEEE International Conference on Communications (ICC '08), pp. 3495-3500, Beijing, China, May 2008.

[11] WINNER, "Final report on identified RI key technologies, system concept, and their assessment," Tech. Rep. D2.10 v. 1.0, WINNER-2003- 507581, December 2005. 
[12] W. Yu, W. Rhee, S. Boyd, and J. M. Cioffi, "Iterative waterfilling for Gaussian vector multiple-access channels," IEEE Transactions on Information Theory, vol. 50, no. 1, pp. 145152, 2004.

[13] M. Sternad, T. Svensson, and G. Klang, "The WINNER B3G system MAC concept," in Proceedings of the 64th IEEEVehicular Technology Conference (VTC '06), pp. 3037-3041, September 2006.

[14] E. Jorswieck, A. Sezgin, B. Ottersten, and A. Paulraj, "Feedback reduction in uplink MIMO OFDM systems by chunk optimization," EURASIP Journal on Advances in Signal Processing, vol. 2008, Article ID 10.1155/2008/597072, 14 pages, 2008.

[15] H. G. Myung, K. J. L. Pan, R. Olesen, and D. Grieco, "Peak power characteristics of single carrier FDMA MIMO precoding system," in Proceedings of the 66th IEEE Vehicular Technology Conference (VTC'07), pp. 477-481, October 2007.

[16] A. Narula, M. D. Trott, and G. W. Wornell, "Performance limits of coded diversity methods for transmitter antenna arrays," IEEE Transactions on Information Theory, vol. 45, no. 7, pp. 2418-2433, 1999.

[17] H. Wu and T. Haustein, "Sum rate analysis of SDMA transmission in single carrier FDMA system," in Proceedings of the 11th IEEE Singapore International Conference on Communication Systems (ICCS '08), pp. 846-850, Guangzhou, China, November 2008.

[18] D. Tse and P. Viswanath, Fundamentals of Wireless Communication, Cambridge University Press, Cambridge, UK, 2005.

[19] D. S. Baum, J. Hansen, G. Del Galdo, M. Milojevic, J. Salo, and P. Kyösti, "An interim channel model for beyond-3G systems: Extending the 3GPP spatial channel model (SCM)," in Proceedings of the 61st IEEE Vehicular Technology Conference (VTC'05), pp. 3132-3136, June 2005.

[20] H. Wu, T. Haustein, and P. A. Hoeher, "On the information rate of single-carrier FDMA using linear frequency domain equalization and its application for 3GPP-LTE uplink," Eurasip Journal on Wireless Communications and Networking, vol. 2009, Article ID 957159, 11 pages, 2009.

[21] 3GPP TS 36.211 and Technical Specification Group Radio Access Network, "Requirements for Evolved UTRA (E-UTRA) and Evolved UTRAN (EUTRAN); Physical Channels and Modulation (Release 8)," September 2009.

[22] V. Chandrasekhar, J. G. Andrews, and A. Gatherer, "Femtocell networks: a survey," IEEE Communications Magazine, vol. 46, no. 9, pp. 59-67, 2008. 